\title{
Sistematización teórica sobre el desarrollo del movimiento de artistas aficionados en las instituciones primigenias $y$ en las universidades cubanas
}

\author{
Milagros de la Caridad Socas Reinoso \\ Universidad Agraria de La Habana | Cuba \\ sara@unah.edu.cu

\section{Glenda Dalmau Gómez} \\ Universidad Agraria de La Habana | Cuba
}

\section{Resumen}

El Movimiento de Artistas Aficionados (MAA) de la Federación Estudiantil Universitaria (FEU), constituye una esfera de actuación, donde el universitario encuentra el espacio no solo para crecerse profesionalmente, sino también de manera cultural. El presente trabajo realiza, por primera vez, una sistematización teórica sobre el desarrollo de este movimiento cultural en las instituciones primigenias y en las universidades cubanas. En particular se analiza el desarrollo y actualidad del MAA en la Universidad Agraria de La Habana (Unah). Se aboga por la materialización del lineamiento número cinco del Programa Nacional de Extensión Universitaria, a través del cual se pretende estrechar la relación existente entre los principios básicos de la Extensión Universitaria y la relación Universidad-CulturaSociedad.

\section{Palabras claves}

Extensión Universitaria, Movimiento de Artistas Aficionados, Comunidad Universitaria. 


\section{INTRODUCCIÓN}

Entre Universidad-Sociedad existe una relación indisoluble, donde la universidad como máxima expresión de institución cultural, tiene la misión de la formación de un profesional, atendiendo a sus procesos sustantivos, referentes a la Docencia cuyo objetivo se centra en preservar la cultura, la investigación, en desarrollarla y la extensión en promover dicha cultura.

Para afianzar esta relación, el estado cubano en su VI Congreso del Partido Comunista de Cuba (PCC), planteó en su Lineamiento \# 163 la necesidad de: “Continuar fomentando la defensa de la identidad, la conservación del patrimonio cultural, la creación artística y literaria y la capacidad para apreciar el arte. Promover la lectura, enriquecer la vida cultural de la población y potenciar el trabajo comunitario como vías para satisfacer las necesidades espirituales y fortalecer los valores sociales" (PCC, 2011, p. 25). Para ello, “[...] se hace de vital importancia la inclusión de una educación artística dentro de los sistemas educacionales cubanos, con el fin de lograr una formación más integral y de hacer resultar a la cultura como los medios técnicos de información de las grandes masas estudiantiles" (HART, 1982).

Las universidades cubanas como instituciones culturales por excelencia y deudoras de la formación de un futuro profesional íntegro y capaz, son los escenarios destinados a la articulación de un trabajo cultural masivo que potencie el desarrollo artístico del Movimiento de Artistas Aficionados (MAA), prestándole un especial interés a las habilidades y capacidades que se desarrollan en los estudiantes y profesores ampliándoles el nivel cultural.

El MAA, constituye uno de los más grandes logros del socialismo en el ámbito de la cultura. Surgiendo como una vía de enorme importancia para propiciar de forma masiva la creación popular en la década de los 60 del siglo XX. Desde sus inicios su política esencial consistió en procurar la organización de grupos de aficionados vinculados a la creación popular y a la promoción de la creación artística. En correspondencia con lo que plantea.

A partir de la reflexión anterior, la Universidad Agraria de La Habana (Unah), hizo gala durante años de un dinámico y efectivo MAA, el cual aportó a la palestra cultural, artistas con una importante trayectoria en el ámbito nacional e internacional. Este 
Sistematización teórica sobre el desarollo del movimiento de artistas aficionados

trabajo presenta una sistematización teórica sobre el desarrollo del MAA en las instituciones primigenias y en las universidades cubanas, siendo de particular atención el desarrollo de este movimiento cultural en la UNAH.

\section{DESARROLLO}

El Movimiento de Artistas Aficionados, no solo constituye un agente de alegría, sino que también es depositario de una responsabilidad, que se evidencia en los niveles de calidad que propone alcanzar con su arte. Un MAA está compuesto por individuos, "[...] con deseos de cultivarse dentro del arte, de formar parte activa del movimiento cultural en cuanto al arte y la literatura de su localidad y nación, de realizarse más plenamente como ser humano, de enriquecer su vida. Lo que lo hace un ciudadano participativo, con conciencia creativa en su tiempo y sobre su propia cultura" (ÁVILA, 2013, p. 56).

De acuerdo con lo planteado por Julio Mitjans ser un artista aficionado: “(...) es parte del proceso hacia la plenitud del ser humano, es un ciudadano participativo, una conciencia hacedora a un tiempo de su propia cultura, auténtico, lúcido, un artista aficionado siempre será una vida más plena, menos manipulable, un sujeto que mira al horizonte convencido de que su huella en pos de la diferencia compartida es única e irrepetible". 1

El artista aficionado tiene su origen en la necesidad plena del ser humano de expresar a través de las artes, la gama de conocimientos y aptitudes aprehendidas, lo que lo hacen enriquecer su espíritu y goce estético. Este, no solo es aquel que cuenta con una potencialidad artística y la desarrolla en pos de lograr su realización personal, sino que el término de artista aficionado, comprende a su vez a aquel individuo que disfruta y percibe el arte como componente de su desarrollo humano.

Aroche (2006, p. 15), por su parte plantea que: “[...] existen dos tipos de aficionados, uno que asume la afición como hobby o pasatiempo para su desarrollo cultural y no depende su afición de disposiciones innatas, sino de motivaciones e intereses culturales; mientras que el otro tipo de aficionado si depende de las aptitudes 
hacia una determinada manifestación artística y literaria, lo cual lo hace tan hábil como un profesional [...]".

El artista aficionado y el MAA, constituyen patrimonio de la comunidad, por lo que es responsabilidad de todas las instituciones, organizaciones y estructuras sociales, establecer las condiciones para el quehacer de estos creadores y portadores originales, encargados de asegurar la salvaguarda de la cultura, que enmarca la preservación del patrimonio cultural vivo, equivalente a la identidad cultural local, regional y nacional.

El MAA, en Cuba tuvo sus comienzos en la década de los 1960. Se tienen referencias de que las primeras organizaciones que promovieron la creación y desarrollo del movimiento de aficionados, se corresponden a las Fuerzas Armadas Revolucionarias (FAR), el Ministerio del Interior (MInint), la Central de Trabajadores de Cuba (CTC), y los pioneros (OPJM) como forma de recreación y aprovechamiento del tiempo libre, logrando la participación activa de las masas.

En 1960 se crearon los primeros grupos de aficionados con obreros y campesinos, y para sistematizar esta labor se fundó en septiembre de ese mismo año el Departamento de Extensión Teatral del Teatro Nacional, adscrito a la Dirección de Cultura del Ministerio de Educación. Los compañeros elegidos para el desarrollo de estas funciones, constituían profesores universitarios encargados de preparar a un grupo de jóvenes que serían los facultados de llevar a cabo el trabajo dirigido fundamentalmente a los campesinos. El inicial objeto de interés fue el teatro. Se formaron grupos teatrales y se organizaron concursos para instructores de la especialidad, posteriormente se abrió el trabajo a la música y la danza.

El ocho de octubre de 1960 se presentó el primer grupo de aficionados al teatro, compuesto por trabajadores del Teatro Nacional con la pieza "La tasa de café". Con la celebración en marzo de 1961 del Festival de Teatro Obrero y Campesino, se incentiva la formación y atención de grupos, solistas, trovadores y coros, creándose así los Centros de Aficionados en las cabeceras provinciales.

A fines de 1961 y comienzos de 1962 se traslada el Departamento de Extensión Cultural del Teatro Nacional para el Consejo Nacional de Cultura y se nombra Departamento de Extensión Cultural, que posibilitó la creación de una Comisión Nacional para el fomento de grupos de aficionados. Esta comisión tendría un personal que se dedicaba individualmente a diferentes sectores poblacionales, correspondientes 
Sistematización teórica sobre el desarollo del movimiento de artistas aficionados

a los estudiantes, Asociación Nacional de Agricultores Pequeños (Anap), FAR, Minint y más tarde una comisión que se dedicaría a atender el movimiento de aficionados, que agrupaba a aquellas personas que poseían limitaciones físicas-motoras.

Con el progresivo desarrollo del MAA, el Departamento de Extensión Cultural, en plena década del 1960, pasó a figurar como la Dirección Nacional de Aficionados. Esta contaría con un cuerpo de especialistas de las diferentes manifestaciones artísticas, encargados de preparar a monitores y activistas de los diferentes organismos y organizaciones para el desarrollo del movimiento en la base.

En el año 1963 se organizan los primeros festivales nacionales, los cuales abarcaban artistas de los diversos sectores. Con el desarrollo y organización del movimiento de aficionados en los organismos y organizaciones, se comienzan a desarrollar festivales individuales en cada una de las instituciones, donde los festivales obreros y universitarios resultaron los de mayor auge, además de los festivales de la OPJM. Llegaron a realizarse Festivales Nacionales de algunos sindicatos. Los festivales de aficionados cobraron tanto auge en el país, que hasta sectores como el de la construcción desarrolló su propio festival.

Para facilitar la participación de los artistas aficionados fue creada la licencia cultural, que autorizaba al artista aficionado a faltar determinado período del año para los eventos nacionales e internacionales.

Antes de producirse la separación del Misterio de Cultura y Educación y a partir del Consejo Nacional de Cultura, fue creado el Centro Metodológico de Aficionados el cual funcionaba anexo a la Dirección Nacional de Aficionados. Este centro se destinó a la orientación técnica del MAA y a la superación para los instructores de arte, cuya misión se centraba en el cultivo y esparcimiento del MAA. Centro que unido a la Dirección Nacional de Aficionados, tomó diferentes nombres, aunque su función continuó siendo la misma. Es así como con el devenir de los años tomó el nombre de Consejo Nacional de Casas de Cultura.

El asumir diferentes nombres no cambió la esencia del trabajo del mismo, orientar, apoyar y desarrollar el MAA, uno de los logros más importantes de la Revolución desde el punto de vista cultural. Por ello dentro de los Objetivos Estratégicos de la institución se plantea: [...] contribuir al crecimiento espiritual y elevación de la calidad de vida de la población cubana mediante la atención a los aficionados al arte y a 
Sistematización teórica sobre el desarollo del movimiento de artistas aficionados

la literatura, la formación de públicos, la atención al Movimiento de Artistas Aficionados y la salvaguarda de la Cultura Popular Tradicional” (CNCC, 2006, p. 4). Logrando así un protagonismo sustancial, en el desarrollo del arte y la literatura, como componentes activos del intelecto humano.

En Cuba, donde crear una cultura del pueblo y para el pueblo ha sido uno de los principios más importantes propugnados desde el 1ro de enero de 1959, y sobre el que se ha trabajado de forma incansable, artistas aficionados son los niños, estudiantes, obreros, profesionales, soldados, discapacitados, amas de casa, cederistas, etc., gracias a las amplias oportunidades que se han ofrecido al pueblo durante todos estos años de Revolución, no solo para aprehender la cultura, sino además para practicarla, sin distinción de raza, sexo, edad o cualquier otra condición social.

Los MAA gozan de gran importancia dentro del desarrollo cultural del país, “constituyen uno de los principales logros de la Revolución cubana en el ámbito de la cultura, en tanto ha logrado la incorporación de todos los sectores sociales a la actividad cultural en las diferentes manifestaciones del arte y la literatura (Ávila, 2013: 56). Considerado como una de las más grandes creaciones culturales del socialismo, los MAA, han representado la solución de los problemas del empleo del tiempo libre de los individuos en la sociedad, como alternativa de una recreación sana, elevación del intelecto y desarrollo de las potencialidades artísticas individuales.

Durante los años 1990, con el llamado Período Especial y el recrudecimiento económico, todas las esferas del país se vieron severamente afectadas. Comenzó a desquebrajarse así, todo cuanto se había logrado en lo correspondiente a los MAA. Lo que ante había sido un pleno goce y desarrollo artístico con la realización de los festivales de aficionados de cada organización de masa, ahora había sido apagado lentamente por la situación de crisis atravesada por el país, sin embargo en los Centros Educacionales a pesar de ser uno de los sectores más abatidos por la situación, los MAA cobraron un mayor auge.

El MAA, dentro de los Centros Educacionales, contó con gran relevancia entre los estudiantes y profesores, pues no solo ayudó a la erradicación del tiempo muerto y a la recreación sana, sino que también constituyó una mejor formación académicaintelectual, donde el nivel cultural de cada estudiante se hacía más amplio y más rico en sí. 
Sistematización teórica sobre el desarollo del movimiento de artistas aficionados

En el ámbito universitario, el MAA tuvo sus inicios mucho antes del triunfo revolucionario. En la década del 40, específicamente en 1941, cuando el teatro en Cuba, no contaba con ningún tipo de apoyo, en la Universidad de La Habana se desarrollaron acciones de carácter artístico-cultural, protagonizadas por importantes figuras de las Artes y las Letras y que dejaron un efecto positivo en el reconocimiento social de la universidad como institución cultural.

Posteriormente se suman la Universidad de Oriente y la Universidad Central de Las Villas, al fundarse en 1947 y 1952, respectivamente. Hasta que por Resolución Rectoral en la Universidad de La Habana, se constituye la Comisión de Extensión Universitaria que, al menos teóricamente, se planteaba como objetivo crear un ambiente propicio al desarrollo del afán de superación espiritual que redundara en el nivel cultural del pueblo.

Con el triunfo revolucionario del 1ro de enero de 1959, el MAA en las universidades, pasó a estar conformado por los alumnos y profesores de cada una de las instituciones rectoras. Se comenzaron a dar los primeros pasos para la implementación de la educación artística dentro de la Educación Superior, pues al decir de Armando Hart: “[...] efectivamente, responde a una necesidad de la formación integral del hombre en el socialismo. Así que se trata del problema de la estética y del arte, de un problema ubicado dentro del campo de educación. No hay educación completa sino hay educación artística" (HART, 1982, p. 6). Por lo que se trataba no solo de la formación del profesional en el ámbito académico, sino también de un profesional totalmente comprometido con su formación dentro de una cultura general integral.

En 1963, se realizaron los primeros Festivales de Artistas Aficionados de la FEU, condicionándose así un sistema de actividades que respondían al desarrollo del movimiento de aficionados, cobrando cada vez más protagonismo dentro de las universidades cubanas. Su accionar provocó que, en 1976 con la creación del Ministerio de Educación Superior (MES) y el Ministerio de Cultura (Mincult), se produjera la ratificación de los Departamentos de Actividades Culturales (antecedente de las Vicerrectorías, Direcciones y Departamentos de Extensión Universitaria) en las universidades y otros centros educacionales, quedando institucionalizado el MAA de la FEU. A partir de esta fecha "el Movimiento de Artistas Aficionados de la FEU (MAA) es 
Sistematización teórica sobre el desarollo del movimiento de artistas aficionados

reconocido por el Ministerio de Cultura, como el de mayor nivel y calidad en el país, avalado por su sistematicidad y proyección social” (GUTIÉRREZ, 2013, p. 23).

A raíz de la ratificación de los Departamentos de Actividades Culturales dentro de la Educación Superior, su visión estuvo centrada en ofrecer una atención análoga a la cultura en la amplia red de centros creados por la Revolución. Sin embargo las principales tareas surgidas de los Departamentos, estuvieron destinadas al desarrollo y proliferación del MAA de la FEU, además de la implementación de la aplicación de los programas relacionados con la Educación Artística, los cuales sufrieron el rechazo y la incomprensión de las instituciones y los estudiantes.

En la intervención realizada por el Ministro de Cultura, en la reunión con los jefes de Departamentos de Actividades Culturales y los dirigentes de la FEU, se plantea: “[...] no debemos escatimar esfuerzos por aumentar y diversificar el Movimiento de Artistas Aficionados. Para ello es indispensable el esfuerzo conjunto de los departamentos de Actividades Culturales, de las direcciones de Cultura Provinciales y municipios, y de la FEU que debe desplegar una labor de masas cada vez más amplia, apoyándose en un fuerte grupo de monitores y actividades que resultan indispensables para poder atender un movimiento artístico en forma realmente masiva" (HART, 1982, p. 8). Se demostró la importancia del MAA y la necesidad de la Educación Artística dentro de los estatutos de la Educación Superior, como máxima gestora de la formación social, académica y cultural de los individuos.

Durante este período, se crean nuevas concepciones sobre el accionar de los Departamentos de Actividades Culturales dentro de los Centros de Educación, lo cual propició, que se convirtieran en los actuales Departamentos de Extensión Universitaria, con nuevas concepciones de trabajo. Se instauró el reglamento para la realización de las actividades de Educación Artística en los estudiantes de la Educación Superior, se fundaron las Comisiones de Extensión en las facultades y centros de atención, que se centraban básicamente en los nuevos objetivos de la esfera cultural de la Educación Superior.

Teniendo en cuenta lo expresado por el entonces Ministro de Cultura Armando Hart, en las universidades de todo el país se intensificó el trabajo para el desarrollo y el reconocimiento del MAA, como máxima expresión de Educación Artística en los Centros Universitarios. En correspondencia (MARTÍN, 1985, p. 34), expresó: “[...] en las 
Sistematización teórica sobre el desarollo del movimiento de artistas aficionados

actividades culturales y la enseñanza de diferentes manifestaciones artísticas del arte que se lleva a cabo, desempeñan un papel importante las organizaciones estudiantiles. Esto contribuye al desarrollo de los estudiantes en la capacidad de percibir, sentir, pensar, analizar y expresar los valores de las diferentes manifestaciones culturales, garantizando con ello su participación activa en la promoción cultural en el medio social donde se desarrollará su actividad profesional, además de una mayor satisfacción personal".

A pesar de todos los cambios sustanciales realizados en las universidades cubanas, con el fin de mejorar la formación professional que se les impartía a los estudiantes, el Sistema de Educación Superior fue altamente criticado en las sesiones del III Congreso del Partido, realizado en 1986. Al referirse sobre la calidad en la formación de los estudiantes de la Educación Superior, en el Informe Central del Congreso quedó plasmado "[...] su desarrollo cultural integral no es satisfactorio" (CONGRESO DEL PCC 1986, p. 10).

Con carácter sistemático, se intensificaron las labores requeridas a la puesta en marcha del "Programa para el Desarrollo Cultural Integral de los Estudiantes Universitarios", con el que se pretendía dar respuesta a la fuerte crítica realizada en el Informe Central al III Congreso del Partido. Durante este año, tuvo lugar la 1ra Jornada Científica del Trabajo Cultural de la Educación Superior, donde el entonces Ministro de Cultura Armando Hart, hacía referencia a las posibilidades y necesidades del país en la esfera de la cultura, enfatizando en el indispensable papel que tienen las universidades en la promoción y extensión cultural.

En los años siguientes se continuó la labor iniciada en las universidades, con el fin de restablecer así los principios básicos de la Educación Artística y la potencialización del MAA, dentro de la formación profesional brindada por la institución, logrando la formación de un futuro profesional capaz de desarrollar capacidades, sensibilidades y habilidades que le permitieran su desarrollo en el ámbito laboral y social. Sin embargo los nuevos embates económicos sufridos por el país, afectaron todo tipo de desarrollo cultural. La Educación Superior Cubana no fue acepta de las condiciones socioeconómicas impuestas al país por diversos factores, que ocasionaron la necesidad de un reordenamiento general de los diferentes sectores de la nación en la etapa denominada Período Especial. 
Sistematización teórica sobre el desarollo del movimiento de artistas aficionados

Durante el denominado Período Especial la actividad cultural en la Educación Superior sufrió un deterioro, debido a la situación y los obstáculos impuestos por las condiciones desfavorables del sistema. Disminuyeron considerablemente los niveles de las actividades culturales, en particular los festivales del MAA de la FEU, sin embargo la existencia de nuevas necesidades espirituales de la población universitaria, principalmente de los estudiantes, propiciaron un crecimiento vertiginoso. Se encontraron alternativas para la creación, donde se realizaron numerosas innovaciones que tuvieron la más alta aceptación y cooperación de las masas estudiantiles. La situación vivida en todas las esferas de desarrollo del país, estimuló el avance progresista del trabajo cultural en las universidades, potencializando la continua existencia y fortalecimiento del MAA.

En la primera década de los años 1990, a pesar de que el MAA de la FEU decayera retrospectivamente, se lograron progresos significativos en el desarrollo de la labor de los artistas aficionados en la Educación Superior. Logrando que hacia 1994 se evidenciara la completa recuperación del MAA en todas sus manifestaciones, restableciéndose así los Festivales a nivel de facultad, centro y provincia. En el mes de octubre de ese mismo año, después de numerosos esfuerzos se logró reanudar la realización de los Festivales Nacionales de Artistas Aficionados de la FEU; evento que no se realizaba desde 1990 perdiendo así, su sistematicidad.

Con el decursar de los años el MAA de la FEU, pasó a figurar como un logro de la Extensión Universitaria, proceso que integra tríptico misional de la Educación Superior cubana: que enuncia la preservación de la cultura mediante la Docencia, su desarrollo mediante la Investigación y su promoción mediante la Extensión Universitaria.

Por otra parte, el Programa Nacional de Extensión Universitaria establece dentro de su lineamiento número cinco la "promoción del fomento de actividades extracurriculares para el desarrollo cultura integral de los estudiantes" (González y González, 2003). En cuyo lineamiento queda registrado la necesidad de mantener y desarrollar los niveles cualitativos del Movimiento de Artistas Aficionados de la FEU (GONZÁLEZ y GONZÁLEZ, 2003), como expresión máxima del desarrollo cultural de la universidad como institución gestora de la formación plena del hombre, a través de la explotación de todas sus potencialidades. 
Sistematización teórica sobre el desarollo del movimiento de artistas aficionados

El nivel formativo del MAA está vinculado al perfeccionamiento de intereses, motivaciones y aptitudes artísticas y literarias y al asesoramiento de los artistas aficionados, al incremento de opciones extracurriculares orientadas al desarrollo de aficiones en los estudiantes que de manera sistemática sean capaces de apreciar, disfrutar y promover la obra cultural, artística y literaria... y el empleo sano y culto del tiempo libre (MES, 2004). Todo MAA, en un contexto universitario debe de estar sustentado en las necesidades y motivaciones de los estudiantes, en su real capacidad de asociarse, comunicarse, actuar o transformarse.

El MAA de la FEU, tiene entre sus principales objetivos crear un ambiente donde los estudiantes no solo estudien, sino también logren la realización de una vida universitaria, estableciendo un medio sano donde se forma el futuro profesional, fortaleciendo el sentido de pertenencia de la organización. Propone además, una forma de utilidad más asequible al tiempo libre de cada uno de los estudiantes universitarios, propiciando un desarrollo de la creación artística del MAA en su total plenitud; al decir de Hart: "La política esencial para el trabajo del Movimiento de Artistas Aficionados no debe ser la de imitar a los grupos y solistas profesionales. Debe de organizar grupos de aficionados vinculados a la creación popular, de modo que se promueva la creación artística. No se trata de la imitación de los profesionales, se trata de impulsar un arte de raíz popular que promueva la creación artística por parte de los aficionados" (HART, 1982, p. 8).

En la actualidad el MAA de la FEU se encuentra institucionalizado y reconocido a niveles ministeriales por parte del Mincult, pero principalmente por el MES, quien establece la necesidad de "[...] incrementar las opciones extracurriculares orientadas al desarrollo de aficiones en los estudiantes a partir de conceptualizar al aficionado como aquel que de manera sistemática sea capaz de apreciar, disfrutar y promover la obra cultural, artística y literaria [...] y el empleo sano y culto del tiempo libre" (MES, 2004).

Con el fin de lograr tales propósitos, en estos nuevos tiempos se ha logrado implementar una serie de acciones que facilitan el desarrollo y avance considerable del MAA. En su desarrollo y consolidación ocupan un rol decisivo las instituciones políticas y culturales asociadas al trabajo cultural, en especial los instructores de arte, provenientes de las Casas de Culturas. Se realizan talleres de apreciación y creación artístico-literarios. Se realizan los Festivales del MAA de la FEU con cierta periodicidad, 
Sistematización teórica sobre el desarollo del movimiento de artistas aficionados

donde la realización de los mismos constituye la máxima expresión del proceso de creación artística.

El MAA constituye en la actualidad, la manera de aprovechar los recursos en bien de la cultura universitaria y del pueblo en sentido general. Su quehacer ha logrado que el arte rebase los teatros, las galerías, los espacios habituales donde se desarrollan de manera ascendentes las más genuinas obras del intelecto humano, para llegar a las comunidades, pero no solo a la comunidad de un municipio determinado, sino a su más grande comunidad universitaria.

Este movimiento responde al espacio de creación, donde los estudiantes de la Educación Superior afloran y emanan de acuerdo con sus intereses y necesidades, la autenticidad propia de su desarrollo académico e intelectual, poniendo en práctica las habilidades adquiridas durante su avance por las diferentes enseñanzas.

Hoy en día, cuando se viven los duros embates de una crisis económica mundial, el MAA de la FEU, constituye un medio de desarrollo para el bien de la comunidad universitaria. Genera variedad de actividades culturales y a su vez contribuye a la formación política, social y cultural, de un profesional formado bajo los postulados de una educación general integral.

Es de vital importancia la atención especializada a los MAA de la FEU, pues de ellos se derivan agrupaciones artísticas relevantes en la esfera universitaria, como lo constituyen Telón Abierto de Ciego de Ávila, Alma Mater de la Universidad de La Habana y el Conjunto Artístico Maraguán de la Universidad de Camagüey. A quienes su loable desempeño los ha ubicado en la cúspide de los MAA del país.

La importancia que se deriva de los MAA, no está solo en la labor que se desprende en las funciones educativas de la universidad, ni en todo cuanto aporta a la formación profesional de los estudiantes, ni tan siquiera al espacio donde se elimina el ocio; el MAA constituye una vía de crecimiento espiritual para sus cultores, para sus espectadores, propicia que se establezcan vínculos afectivos entre sus miembros, genera competencias para la vida y transforma el proyecto de vida de sus integrantes.

Es una realidad que Cuba, agradece al MAA, la trayectoria artística de importantes representantes de la cultura nacional en todas las manifestaciones artísticas, quienes además reconocen cuánto le aportó su tránsito por el movimiento para los éxitos de su actividad profesional. Merecen la pena mencionarse: Alejandro 
Sistematización teórica sobre el desarollo del movimiento de artistas aficionados

García (Virulo), Jorge Gómez (Director de Moncada), Manuel Calviño, (psicólogo y comunicólogo), Luis Silva y Adrián Berazaín.

En la Unah, una de las problemáticas que acarrea frecuentemente el debate, lo constituye el desarrollo del MAA, que representa todo un reto para la comunidad universitaria. Dentro de este escenario se articulan acciones extracurriculares, mediante las cuales se complementa la formación de los futuros profesionales que egresan de la institución, haciéndose mayor énfasis en las acciones promocionales correspondientes a la salud y a la cultura.

Esta última encuentra su materialización en el MAA desde sus formas organizativas, de acuerdo con lo establecido en el Programa Nacional de Extensión Universitaria donde se establece, "promover el fomento de actividades extracurriculares para el desarrollo cultural integral de los estudiantes” (GONZÁLEZ y GONZÁLEZ, 2003, p. 6). La universidad contaba con un fuerte y próspero MAA, “donde los espacios de interacción se encontraban establecidos, logrando una motivación en los estudiantes a pertenecer y ser parte del movimiento de aficionados de la universidad en general" (GONZÁLEZ, 2014). Se organizaban galas culturales, donde se producía un intercambio entre los estudiantes extranjeros y los estudiantes cubanos, encontrándose puntos de contacto entre una cultura y otra. Existía un grupo de danza, que conformaba la comparsa de la universidad que gozaba de gran reconocimiento en la institución y en diversas ocasiones la representó en eventos culturales de gran envergadura.

La parte musical, se componía de estudiantes de las diferentes carreras que sentían una fuerte afición por la música, logrando que de "adentro de sus filas surgieran grupos y cantantes de las agrupaciones que hoy día conforman la élite cultural cubana, tal es caso de Yurumí y sus Hermanos y de la solista de la agrupación Azúcar Negra (CAPÓ, 2014). Contaba, además, con un grupo de teatro, que bajo el nombre de Los Pinos Nuevos que participaba en los diferentes eventos culturales de la organización estudiantil, resultando uno de los más reconocidos.

Los aires que se respiraban por aquella época resultaban inauditos para muchos, pues nadie lograba explicarse como una universidad enclavada en el Km 23 y medio de la Autopista Nacional, alejado de los grandes Centros Culturales, podía fomentar y mantener un MAA con tanta fuerza. Su secreto se enmarcaba en el protagonismo e iniciativas de sus estudiantes, que hacían de la comunidad universitaria el escenario de 
Sistematización teórica sobre el desarollo del movimiento de artistas aficionados

desarrollo de numerosas actividades que contribuían a la formación de un fuerte movimiento.

En la actualidad, se han impulsado acciones para lograr un mayor reconocimiento de los aficionados, pues si en la antigüedad para cada una de las actividades, ya fuesen de carácter político o cultural, se invitaban artistas aficionados de otros centros como la Asociación de Hermanos Saiz, en la actualidad se opta por amenizar las actividades con la participación de los aficionados de la Unah, creándoles puntos de interacción donde puedan lograr expresar su arte, como ejemplos fehacientes: Talleres de Valores, conmemoración de efemérides, y proyectos en la Residencia.

Cultura Provincial y el Departamento de Extensión Universitaria, han promovido talleres de creación artística, donde el aficionado encuentra el espacio para desarrollar y explotar su potencial artístico. Se han incrementado los niveles de motivación y el número de estudiantes que participan como miembros del movimiento. Se desarrollan proyectos investigativos y de transformación, en pos de detectar cuáles son las causas y las posibles soluciones para incentivar la participación del estudiantado en actividades de esta índole. Además se realizan "labores de promoción y alianzas estratégicas con diversas instituciones culturales de la provincia y el país (Centro Nacional de Danza; Proyecto Cubanos en la Red; Cultura provincial, Dpto. de Audiovisuales, Librerías y de Artes Milenarias)" (BLANCO, 2013) con el fin de lograr un accionar más objetivo del MAA.

Teniendo en cuenta, lo anteriormente planteado se debe reconocer que institucionalmente se impulsan diversas actividades en pos de lograr un impulso del MAA y su constante transformación; se pretende involucrar con responsabilidad a cada una de las facultades para que cumplan con lo establecido en términos de convocatoria a los festivales y potenciar acciones que lejos de desacreditar al movimiento, le den una mejor estructuración y finalidad.

Con el logro de todas estas acciones la universidad logra percibir al movimiento de aficionados en el futuro, como el espacio donde el aficionado se crezca culturalmente, formándose bajos los preceptos de la cultura general integral, que promociona y viabiliza el Sistema de Educación Cubana. Además de verlo como la actividad que facilita la educación en valores e incrementa el sentido de identidad de los estudiantes en la institución. 


\section{CONCLUSIONES}

- El trabajo realizado constituye un primer acercamiento a la sistematización histórica del desarrollo del Movimiento de Artistas Aficionados en las universidades cubanas.

- El Movimiento de Artistas Aficionados ha contribuido a la trayectoria artística de importantes representantes de la cultura nacional en todas las manifestaciones artísticas.

- Es importante fortalecer la organización del Movimiento de Artistas Aficionados en la Unah, para lograr elevar la cultura y el desarrollo integral del profesional que se forma en esta casa de altos estudios.

\section{REFERENCIAS}

AROCHE, A. Desarrollo cultural. Plataforma para el debate, en: Congreso Internacional de la Educación Superior. Universidad 2006. VIII Taller de Extensión Universitaria. Cuba: MES. 2006,p. 15.

ÁVILA, F. El Movimiento de Artistas Aficionados: nuestra razón de ser. La Habana. MINCULT. 2013,p. 56

BLANCO, A. Testimonio Oral sobre "Acciones para el desarrollo del MAA de la UNAH".2013.

CAPÓ, M. E. Testimonio Oral sobre el “Desarrollo del MAA en la UNAH”. 2014.

CNCC. Objetivos Estratégicos del Consejo Nacional de Casas de Cultura. La Habana [s.n]. 2006,p.4.

GONZÁLEZ, G. Testimonio Oral sobre los “Antecedentes del MAA en la UNAH”. 2014.

GONZÁLEZ, G. Y GONZÁLEZ, M. Extensión: Opción viable en el contexto educativo contemporáneo. La Habana, Cuba: Ciencias Sociales. 2003, p. 69.

GUTIÉRREZ, G. Importancia del Festival para la comunidad universitaria. La Habana. [s.n]. 2013, p. 23.

HART, A. Reunión con los jefes de Departamentos de Actividades Culturales de las Centros de Educación Superior y con los dirigentes de la FEU. La Habana. 1982, [s.n] p.2. 
Sistematización teórica sobre el desarollo del movimiento de artistas aficionados

MARTÍN, E. Situación del Movimiento Estudiantil. Servicios a Estudiantes. La Habana. $1985[$ s.n].

MÊS. Programa Nacional de Extensión Universitaria. Ciudad de La Habana: Ministerio de Educación Superior. 2004.

PARTIDO COMUNISTA DE CUBA. Informe Central del III Congreso del PCC. La Habana. [s.n]. 1986, p.10.

PARTIDO COMUNISTA DE CUBA. Lineamiento de la política, económica y social del Partido y la Revolución. La Habana: 6to Congreso PCC. 2011, p. 25. 
Theoretical systematization on the development of the fond of artists in primary institutions and Cuban universities

\section{ABSTRACT:}

The Movimiento de Artistas Aficionados (MAA - Movement of Fond Artists) of the Federación Estudiantil Universitaria (FEU - Federation Student University student), constitutes a performance sphere, where the university student finds the non alone space to be grown professionally, but also in a cultural way. The present work carries out, for the first time, a theoretical systematizing on the development of this cultural movement in the institutions primigenias and in the Cuban universities. In particular it is analyzed the development and present time of the MAA in the Agrarian University of Havana (Unah). One pleads for the materialization of the limit number five of the National Program of University Extension, through which is sought to narrow the existent relationship between the basic principles of the University Extension and the relationship University-Culture-Society.

\section{Key Words:}

University Extension, Movement of Amateur Artists, University Community

\begin{abstract}
Sistematização teórica sobre o desenvolvimento do movimento de artistas amadores nas instituições primárias e nas universidades cubanas
\end{abstract}

\section{RESUMO:}

0 Movimento de Artistas Amadores (MAA) dos estudantes da Federação Estudantil Universitária (FEU), que constitui uma esfera de atuação onde o estudante universitário encontra o espaço não só para se desenvolver profissionalmente, mas também de forma cultural. 0 trabalho realiza, pela primeira vez, uma sistematização teórica sobre o desenvolvimento deste movimento cultural nas primeiras instituições que o acolheram e nas universidades cubanas. Em particular, analisa 0 desenvolvimento e a atualidade do MAA na Universidade Agrária de Havana (Unah). Parte da linha de número cinco do Programa Nacional de Extensão Universitária, através do qual se busca estreitar a relação existente entre os princípios básicos da Extensão Universitária e a relação Universidade-Cultura-Sociedade.

Palavras-chave: Extensão Universitária, Movimento de Artistas Amadores, Comunidade Universitária

Original submetido em: 26 out. 2015

Aceito para publicação em: 14 nov. 2016

Sobre as autoras:

Milagros de la Caridad Socas Reinoso

Universidad Agraria de La Habana | Cuba

sara@unah.edu.cu

\section{Glenda Dalmau Gómez}

Universidad Agraria de La Habana | Cuba 